\title{
Determination of Enzyme/Substrate Specificity Constants Using a Multiple Substrate ESI-MS Assay
}

\author{
Na Pi and Julie A. Leary \\ Department of Chemistry, University of California at Berkeley, Berkeley, California, USA
}

\begin{abstract}
The traditional method used to investigate the reaction specificity of an enzyme with different substrates is to perform individual kinetic measurements. In this case, a series of varied concentrations are required to study each substrate and a non-regression analysis program is used several times to obtain all the specificity constants for comparison. To avoid the large amount of experimental materials, long analysis time, and redundant data processing procedures involved in the traditional method, we have developed a novel strategy for rapid determination of enzyme substrate specificity using one reaction system containing multiple competing substrates. In this multiplex assay method, the electrospray ionization mass spectrometry (ESI-MS) technique was used for simultaneous quantification of multiple products and a steady-state kinetics model was established for efficient specificity constant calculation. The system investigated was the bacterial sulfotransferase NodH (NodST), which is a host specific nod gene product that catalyzes the sulfate group transfer from 3'phosphoadenosine 5'-phosphosulfate (PAPS) to natural Nod factors or synthetic chitooligosaccharides. Herein, the reaction specificity of NodST for four chitooligosaccharide acceptor substrates of different chain length (chitobiose, chitotriose, chitotetraose, and chitopentaose) was determined by both individual kinetic measurements and the new multiplex ESI-MS assay. The results obtained from the two methods were compared and found to be consistent. The multiplex ESI-MS assay is an accurate and valid method for substrate specificity evaluation, in which multiple substrates can be evaluated in one assay. (J Am Soc Mass Spectrom 2004, 15, 233-243) (c) 2004 American Society for Mass Spectrometry
\end{abstract}

$\mathrm{R}$ ecently, substrate specificity investigations along with specificity constant $\left(k_{c a t} / K_{M}\right)$ measurements have provided significant insights into a number of biologically important enzymes [1-15]. Specificity studies of an enzyme for different natural or synthetic substrates can help determine the enzyme structure and physiological functions $[1,4-7]$ as well as address the key residues involved in substrate binding and catalysis $[2,8]$. Specificity studies can also help to unravel the reaction pathway or catalytic mechanism [9], and guide substrate structure-based inhibitor development and drug design $[3,10,11]$. For example, Hara et al. examined the specificity of three isoenzymes of human 3(20) $\alpha$-hydroxysteroid dehydrogenase and successfully addressed their roles in neurosteroid metabolism [1]. Griffith and coworkers evaluated the influence of divalent metal ions on the substrate specificity of $\gamma$-glutathione synthetase, elucidating the enzyme substrate binding interactions [2]. The novobiocic acid noviosyl transferase activity of NovM and its specificity for

Published online January 1, 2004

Address reprint requests to Dr. J. A. Leary, Department of Chemistry, University of California at Berkeley, 23 Lewis Hall, Berkeley, CA 94720, USA. E-mail: leary@socrates.berkeley.edu various substrate analogs in designing novel coumarinbased antibiotics was recently characterized by Walsh and coworkers [3]. In addition, efforts have been made to alter enzymes by site-directed mutagenesis in order to manipulate substrate specificity [12-14] or enhance enzyme catalysis [15].

Traditionally, the general method of evaluating the specificity of a given enzyme is to measure the specificity constant of a group of substrates individually followed by comparison of measured values, regardless of the assay used for measurement [1-15]. This requires large amounts of material and considerable effort to study substrate libraries. Consequently, a simple and rapid methodology for evaluating enzyme specificity and obtaining $k_{\text {cat }} / K_{M}$ values would be of great interest for kinetic analyses and would dramatically expediate the process of understanding biologically important enzymatic systems.

Recently, the potential of mass spectrometry in studying enzyme catalysis has been widely demonstrated [16-29]. The development of soft ionization techniques, such as electrospray ionization (ESI) and matrix-assisted laser desorption (MALDI), has made mass spectrometry an excellent complementary technique to conventional spectrophotometric methods for 
studying enzyme kinetics [23-29]. With the improvements of resolving power, sensitivity, and versatility, mass spectrometry has become a highly competitive analytical method for detection and characterization of biochemical reaction intermediates that can be used to elucidate enzymatic reaction pathways and catalytic mechanisms [16-22]. Esaki et al. reported a novel catalytic mechanism for L-2-Haloacid dehalogenase involving a cyanoalanine intermediate revealed by LC/MS monitoring of the enzymatic reaction [16]. Likewise, Leary and coworkers reported the identification of a sulfated NodH sulfotransferase (NodST) intermediate formed in a hybrid Ping-Pong mechanism using Fourier transform ion cyclotron resonance (FTICR) mass spectrometry [17]. A facile and broadly applicable ESI-MS assay developed by Leary and coworkers has been used for kinetic analyses on several enzyme systems such as glutathione S-transferase (GST) [27], hexokinase [28], and NodH sulfotransferase (NodST) [29]. The mass spectrometry assay is especially useful because traditional spectrophotometric assays are not applicable to NodST kinetics since no shift in absorption accompanies sulfuryl group transfer. Using this assay, precise and accurate kinetic data were generated for all the enzymes above, and for the first time, a hybrid Ping-Pong Bi-Bi mechanism was unraveled for NodST [17]. A distinct feature of the ESI-MS assay is that product quantification is realized by monitoring the product ion in relation to an internal standard ion in a quenched reaction solution, allowing different product ions of different mass to charge ratio to be monitored and quantified simultaneously in one reaction system. Consequently, development of a method to calculate the specificity constants of multiple substrates based on the concentrations of multiple products in one reaction system would make it feasible to utilize the ESI-MS technique, thus avoiding the redundant "oneat-a-time" measurements in the traditional method. The ability of mass spectrometric assays to monitor many reaction products in one sample makes the investigation of multiple substrate kinetics fast and easy, something that is difficult or even impossible by spectrophotometric assays.

The multiplex ESI-MS assay method reported herein demonstrates a successful combination of the steadystate kinetics calculation model and the ESI-MS multiple product quantification technique for the streamlined specificity constant measurements of multiple competing substrates in one reaction. It is rather unique in that only one reaction system and one ESI-MS measurement are required to obtain the enzyme specificity information for multiple substrates. The validity of this approach was tested on the specificity study of the bacterial carbohydrate sulfotransferase, NodH (NodST) for a library of four chitooligosaccharide substrates.

NodH is one of the host specific nod gene products that is necessary for diverse modification of the core lipochitooligosaccharide (Nod factor) structure in Rhi- zobium meliloti [30-34]. As a GlcNAc-6-O-carbohydrate sulfotransferase, NodH catalyzes the transfer of a sulfuryl group from 3 '-phosphoadenosine 5 '-phosphosulfate (PAPS) to the reducing terminal 6-O position of the Nod factor [30,31]. The presence of the sulfate group is critical for biological activity on the host plant alfalfa, while its absence renders the Nod factor active on the non-host plant vetch [31]. Therefore, the sulfotransferase activity of NodST is a major determinant of host specificity and much of the focus of recent research has been on the substrate specificity of this enzyme [30, 31, 35, 36]. Employing a thin layer chromatography assay, Long et al. [30] and Kondorosi and coworkers [31] discovered that NodST can sulfate biosynthetic chitooligosaccharides in addition to natural Nod factors, although with a lower reaction activity. Kinetic analyses with both Nod factors and chitooligosaccharides of different chain lengths revealed the highest binding affinity and best reaction activity of NodH for the appropriate tetrameric substrates, which guarantees the modification of the tetrameric Nod factor-the most active one in Rhizobium meliloti, and thus contributes to host specificity [31, 37]. Kinetic constants for various chitooligosaccharides have been determined individually by Kondorosi and coworkers [31] and Wong et al. [37], which indicated that chitotetraose is a better substrate than chitotriose, chitopentaose, or chitohexaose, while NodST's activity for chitobiose is more than one order of magnitude lower than that for the other four.

In this study, NodST's specificity for four biosynthetic chitooligosaccharide substrates was investigated. Despite different oligosaccharide chain length, all four acceptor substrates accommodate the transferred sulfate group using the reducing end GlcNAc residue, as shown in Scheme 1. Their specificity constants were determined first using individual kinetic measurements and then by the newly-developed multiplex assay. The results of the two methods were found to be consistent with each other as well as with those reported in the literature, thus demonstrating that the multiplex ESI-MS assay is a valid method for substrate specificity studies for NodST. We believe the simplicity and rapidity of this assay will make it a viable alternative to the more commonly used individual assays.

\section{Experimental}

\section{General Materials and Methods}

All chemical reagents were obtained from commercial suppliers and used without further purification. NodST, chitobiose, and chitopentaose were purchased from Calbiochem (San Diego, CA). Chitotriose and chitotetraose were purchased from Seikagaku America Inc. (Iamsville, MD). All the other compounds were purchased from Sigma Co. (St. Louis. MO), including 3'-phosphoadenosine 5'-phosphosulfate (PAPS), 3'phosphoadenosine $5^{\prime}$-phosphate (PAP) and $\alpha$ - $\Delta \mathrm{UA}$ - 


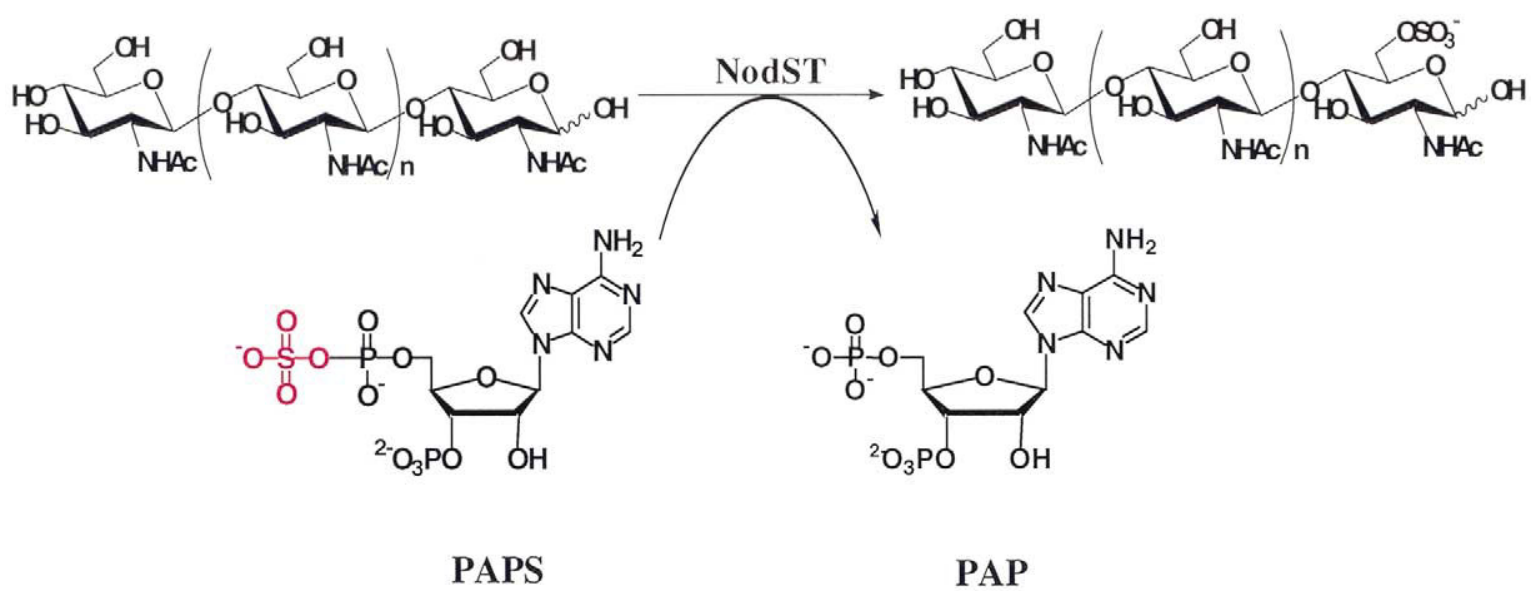

Scheme 1. NodST catalyzes the sulfation of chitooligosaccharide, generating PAP and chitooligosaccharide-6- $\mathrm{OSO}_{3}^{-}$as products. (Chitobiose, $n=0$; chitotriose, $n=1$; chitotetraose, $n=2$; chitopentaose, $n=3$.)

$[1 \rightarrow 3]$-GalNAc-6S $\left(\Delta \mathrm{D}_{\mathrm{i}}-6 \mathrm{~S}\right)$. Commassie plus protein assay reagent $(950 \mathrm{~mL})$ and albumin standard $(10 \times$ $1 \mathrm{~mL}$ ) were purchased from Pierce (Rockford, IL). All the mass spectrometric measurements were performed at $22{ }^{\circ} \mathrm{C}$ in $10 \mathrm{mM} \mathrm{NH}_{4} \mathrm{OAc}(\mathrm{pH}=8.0)$ (Buffer A).

\section{Instrumentation}

A Finnigan LCQ ion trap mass spectrometer equipped with an ESI source and a HPLC pump (ThermoFinnigan, San Jose, CA) was used in this study. The capillary temperature and the spray voltage were kept at $200{ }^{\circ} \mathrm{C}$ and $3.2 \mathrm{kV}$, respectively. Approximately $20 \mu \mathrm{L}$ of each sample solution was injected through a $5 \mu \mathrm{L}$ injection loop and delivered via a $\mathrm{LC}$ pump at a flow rate of $20 \mu \mathrm{L} / \mathrm{min}$. Four product ions $(\mathrm{m} / \mathrm{z} 503,706,909,1112)$ and the internal standard $\left(\Delta \mathrm{D}_{\mathrm{i}}-6 \mathrm{~S}\right)$ ion $(\mathrm{m} / \mathrm{z} 458)$ were monitored in the negative ion mode using selected ion monitoring (SIM). The signals for the ions of interest were optimized by using the automatic tuning option on the instrument. The optimized conditions were then applied in subsequent experiments. When the signal intensity for one sample decreased from approximately $5 \times 10^{5}$ detector counts per scan to $5 \times 10^{3}$ detector counts per scan, indicating the consumption of the former injection, the next sample was introduced. The total ion chromatogram of the Qual Browser program was used to monitor the processing of the sample versus time. An average of $17 \times 3=51$ scans were taken to obtain a spectrum list for each sample, which provides the absolute intensities for the monitored ions along with the relative abundance. The sums of the intensities within 0.8 mass units around the center of each product ion and the internal standard ion were used to determine the intensity ratio $\left(\mathrm{I}_{\mathrm{P}} / \mathrm{I}_{\mathrm{IS}}\right)$.

\section{Individual Kinetic Measurements}

Methodology for product quantification. A stock solution of NodST (200 nM) (NodST stock 1) was prepared in $10 \mathrm{mM} \mathrm{NH}_{4} \mathrm{OAc}(\mathrm{pH}=8.0)$ (Buffer A). Stock solutions of chitobiose $(250 \mu \mathrm{M})$, chitotriose $(250 \mu \mathrm{M})$, chitotetraose $(250 \mu \mathrm{M})$, chitopentaose $(250 \mu \mathrm{M})$, and PAPS $(750 \mu \mathrm{M})$ were prepared in Buffer A. A prereaction solution (total volume $=25 \mu \mathrm{L}$ ) for each of the chitooligosaccharides was prepared by mixing 5 $\mu \mathrm{L}$ PAPS stock solution $(750 \mu \mathrm{M})$ with $5 \mu \mathrm{L}$ chitooligosaccharide stock solution $(250 \mu \mathrm{M})$ and $15 \mu \mathrm{L}$ Buffer A. All four reactions were initiated upon addition of $25 \mu \mathrm{L}$ of NodST stock 1 . The concentrations of PAPS and chitooligosaccharide in each reaction system were $75 \mu \mathrm{M}$ and $25 \mu \mathrm{M}$ respectively, along with $100 \mathrm{nM}$ NodST. The four samples were kept at rt overnight and the enzymatic reaction was allowed to proceed to completion. At this point, the acceptor substrate in each reaction system had been completely consumed as evidenced by the absence of a peak corresponding to the chitooligosaccharide in the ESI-MS spectra, providing a sulfated-chitooligosaccharide concentration of $25 \mu \mathrm{M}$. A $20-\mu \mathrm{L}$ aliquot of each reaction solution was quenched in $80 \mu \mathrm{L}$ of $\mathrm{MeOH}$ containing $6.25 \mu \mathrm{M}$ internal standard, $\Delta \mathrm{D}_{\mathrm{i}}-6 \mathrm{~S}$ (Figure 1a). The resulting four quenched sample solutions were analyzed by selected ion monitoring (SIM) to obtain the single-point normalization factors (see below) for the four sulfated chitooligosaccharide products (chitobiose-6-OSO $\mathrm{O}_{3}^{-}$, chitotriose-6- $\mathrm{OSO}_{3}^{-}$, chitotetraose-6- $\mathrm{OSO}_{3}^{-}$, and chitopentaose-6- $\mathrm{OSO}_{3}^{-}$) (Figure $1 \mathrm{~b}$ ). The four single-point normalization factors were subsequently used for product quantification. 
The single-point normalization factor $(R)$ is determined from eq 1 , through which the relative ion intensity ratio of the product and the internal standard $\left(\mathrm{I}_{\mathrm{P}} / \mathrm{I}_{\mathrm{IS}}\right)$ is related to the ratio of their concentrations. $\mathrm{R}$ can be obtained by analyzing a mixture of the internal standard and the product of known concentration [2729].

$$
\mathrm{R}=\left(\mathrm{I}_{\mathrm{P}} / \mathrm{I}_{\mathrm{IS}}\right) /([\text { Product }] /[\text { Internal standard }])
$$

In this kinetic study, a chondroitin disaccharide, $\alpha$ - $\Delta$ UA-[1 $\rightarrow 3$ ]-GalNAc-6S $\left(\Delta \mathrm{D}_{\mathrm{i}}-6 \mathrm{~S}\right)$ was chosen as an internal standard. The linear response of the $\mathrm{R}$ factor was verified by measuring the ion intensity ratio for various concentrations of the product and internal standard (see "supporting information"). To obtain the single-point normalization factor for each product, two ions were monitored: the internal standard ion, $\left[\Delta \mathrm{D}_{\mathrm{i}}-6-\right.$ $\mathrm{S}-\mathrm{H}]^{1-}$, at $m / z 458$ and the corresponding product ion, which is [chitobiose-6-OSO $]^{1-}$ at $m / z 503$, [chitotriose$\left.6-\mathrm{OSO}_{3}\right]^{1-}$ at $\mathrm{m} / \mathrm{z}$ 706, [chitotetraose-6-OSO$]_{3}{ }^{1-}$ at $\mathrm{m} / \mathrm{z}$ 909 , or [chitopentaose-6-OSO $]^{1-}$ at $m / z 1112$.

For each sample analyzed in the subsequent kinetic measurements, the product concentration can be calculated via eq 2 using the ESI-MS data $\left(\mathrm{I}_{\mathrm{P}} / \mathrm{I}_{\mathrm{IS}}\right)$ and the normalization factor $\mathrm{R}$ determined above.

$$
[\text { Product }]=\left(\mathrm{I}_{\mathrm{P}} / \mathrm{I}_{\mathrm{IS}}\right) \times[\text { Internal standard }] / \mathrm{R}
$$

Kinetic constant $\left(K_{M}, k_{\text {cat }}\right.$, and $\left.k_{\text {cat }} / K_{M}\right)$ determination. A stock solution of NodST (100 nM) (NodST stock 2) was prepared in Buffer A. For each of the four acceptor substrates studied, a series of seven pre-reaction solutions were prepared by mixing the PAPS stock solution $(250 \mu \mathrm{M})$, chitooligosaccharide stock solutions $(0.5,1,2$, 3, 4, 6, $8 \mathrm{mM}$ for chitobiose; 50, 100, 200, 300, 400, 600, $800 \mu \mathrm{M}$ for chitotriose, chitotetraose, and chitopentaose) in Buffer A. After initiating each reaction with 25 $\mu \mathrm{L}$ of NodST stock 2 , the chitooligosaccharide concentrations in the seven reaction solutions were 50 to 800 $\mu \mathrm{M}$ for chitobiose, and 5 to $80 \mu \mathrm{M}$ for chitotriose, chitotetraose, and chitopentaose, while the concentration of PAPS and NodST in each reaction system was 25 $\mu \mathrm{M}$ and $50 \mathrm{nM}$ respectively. The reaction was allowed to proceed for $10 \mathrm{~min}$ for chitobiose, and $4 \mathrm{~min}$ for chitotriose, chitotetraose, and chitopentaose, after which a $20-\mu \mathrm{L}$ aliquot of each reaction solution was quenched in $80 \mu \mathrm{L}$ of $\mathrm{MeOH}$ with $6.25 \mu \mathrm{M}$ internal standard $\left(\Delta D_{i}-6 S\right)$. The seven quenched samples were analyzed by ESI-MS and the amount of product in each sample was quantified using the corresponding singlepoint normalization factor. The $K_{M}, k_{\text {cat }}$, and $k_{\text {cat }} / K_{M}$ values for each chitooligosaccharide were determined by plotting the calculated initial velocity versus chitooligosaccharide concentration using the GraFit program (Version 4.0.12, from Erithacus Software Ltd., Horley, Surrey, UK). Error in these calculations was determined from four replicate experiments.

\section{Substrate Specificity Evaluation Using the Multiplex Approach}

Methodology for product quantification. Stock solutions of chitobiose $(1 \mathrm{mM})$, chitotriose $(1 \mathrm{mM})$, chitotetraose $(1 \mathrm{mM})$, chitopentaose $(1 \mathrm{mM})$, and PAPS (1.5 $\mathrm{mM}$ ) were prepared in Buffer A. Four individual pre-reaction solutions $(50 \mu \mathrm{L})$ for the four acceptor substrates were prepared by mixing $10 \mu \mathrm{L}$ PAPS stock solution and $30 \mu \mathrm{L}$ Buffer A with $10 \mu \mathrm{L}$ chitobiose, chitotriose, chitotetraose and chitopentaose stock solution respectively. Each reaction was initiated with the addition of $50 \mu \mathrm{L}$ of NodST stock 1 . The concentrations of PAPS and chitooligosaccharide in each reaction system were $150 \mu \mathrm{M}$ and $100 \mu \mathrm{M}$ respectively, along with $100 \mathrm{nM}$ NodST. The samples were kept at $\mathrm{rt}$ overnight to allow the enzymatic reactions to proceed to completion. As evidenced by the absence of a peak corresponding to the chitooligosaccharide in the ESI-MS spectra, the acceptor substrate in each reaction system had been completely converted into the corresponding sulfated chitooligosaccharide product, giving a product concentration of $100 \mu \mathrm{M}$. At this point, $75 \mu \mathrm{L}$ of each completed reaction solution were mixed together, generating a $300 \mu \mathrm{L}$ solution mixture (Solution 1) in which the concentrations of the four sulfated chitooligosaccharide products: chitobiose-6- $\mathrm{OSO}_{3}^{-}$, chitotriose-6- $\mathrm{OSO}_{3}^{-}$, chitotetraose-6- $\mathrm{OSO}_{3}^{-}$, and chitopentaose-6- $\mathrm{OSO}_{3}^{-}$were all $25 \mu \mathrm{M}$. Solution 1 was subsequently diluted with Buffer A in seven different ratios, generating Solutions 2 through 8 , in which the concentrations of the four products are $20 \mu \mathrm{M}, 15 \mu \mathrm{M}$, $10 \mu \mathrm{M}, 5 \mu \mathrm{M}, 2.5 \mu \mathrm{M}, 1 \mu \mathrm{M}$, and $0.5 \mu \mathrm{M}$ respectively. A $20-\mu \mathrm{L}$ aliquot of each solution (1 through 8) was quenched in $80 \mu \mathrm{L} \mathrm{MeOH}$ containing $6.25 \mu \mathrm{M}$ internal standard, and the resulting series of eight quenched sample solutions was used to construct the corresponding calibration curves for the four sulfated products.

Selected ion monitoring (SIM) analysis was performed on each of the eight quenched sample solutions. In this particular study, the five ions were monitored simultaneously, the four sulfated products ([chitobiose6- $\left.\mathrm{OSO}_{3}\right]^{1-}$, [chitotriose-6-OSO $]^{1-}$, [chitotetraose-6$\left.\mathrm{OSO}_{3}\right]^{1-}$, and [chitopentaose-6-OSO $\left.{ }_{3}\right]^{1-}$ ) at $m / z 503,706$, 909 , and 1112, respectively, and the internal standard $\left(\left[\Delta \mathrm{D}_{\mathrm{i}}-6 \mathrm{~S}-\mathrm{H}\right]^{1-}\right)$ at $m / z 458$. Consequently, four relative ion intensity ratios were obtained for each quenched sample solution, which fall between the internal standard ion and each of the four sulfated-product ions. For each sulfated product, a calibration curve was generated by plotting the ion intensity ratios of the product and the internal standard $\left(\mathrm{I}_{\mathrm{P}} / \mathrm{I}_{\mathrm{IS}}\right)$ versus their concentration ratios. The slope of each calibration curve (normalization factor) was used for quantification of the product of each competing substrate in one reaction system. 
Specificity constant $\left(k_{\text {cat }} / K_{M}\right)$ measurement and substrate specificity evaluation. A $50-\mu \mathrm{L}$ pre-reaction solution containing multiple chitooligosaccharide substrates was prepared by mixing $10 \mu \mathrm{L}$ PAPS stock solution (250 $\mu \mathrm{M})$, and the four chitooligosaccharide stock solutions (10 $\mu \mathrm{L}$ each) ( $3 \mathrm{mM}$ for chitobiose, and $300 \mu \mathrm{M}$ for chitotriose, chitotetraose, and chitopentaose). After initiating each reaction with $50 \mu \mathrm{L}$ of NodST stock 2 , the chitooligosaccharide concentrations in the multi-substrate reaction solution were $300 \mu \mathrm{M}$ for chitobiose and $30 \mu \mathrm{M}$ for chitotriose, chitotetraose, and chitopentaose, while the concentration of PAPS and NodST in each reaction system was $25 \mu \mathrm{M}$ and $50 \mathrm{nM}$ respectively. At $2,4,6$, and $8 \mathrm{~min}$, a $20-\mu \mathrm{L}$ aliquot of reaction solution was quenched in $80 \mu \mathrm{L}$ of $\mathrm{MeOH}$ containing the internal standard. Upon analysis by ESI-MS, the four sulfated chitooligosaccharide products in each quenched sample were quantified using the normalization factors determined above and the conversion of reaction was plotted versus the reaction time to generate a reaction progress curve. The relative reaction specificity of the four substrates, represented by the ratios of their individual specificity constant $\left(k_{c a t} / K_{M}\right)$, was obtained using their product concentration ratios at $2 \mathrm{~min}$. Furthermore, the value of the specificity constant for each substrate can be calculated based on the determined ratios and the $k_{c a t} / K_{M}$ value of one of the substrates obtained in the individual kinetic measurement.

\section{Results and Discussion}

\section{Individual Kinetic Measurements}

Before the individual kinetic measurement for each substrate, several preliminary experiments with a limited number of chitooligosaccharide concentrations were performed to determine the proper substrate concentration range and quench time. As revealed by the preliminary experiments, chitobiose is the weakest binder to NodST and its $K_{M}$ value is expected to be much higher than that of the other three chitooligomers. Therefore, a substrate concentration range of 50 to 800 $\mu \mathrm{M}$ was used for the kinetic measurement of chitobiose, which is one order of magnitude higher than that used for the other three chitooligomers (5 to $80 \mu \mathrm{M}$ ). Additionally, the chitobiose reaction was allowed to proceed for $10 \mathrm{~min}$ compared to $4 \mathrm{~min}$ for chitotriose, chitotetraose, and chitopentaose, since chitobiose reacts much slower than the other three acceptor substrates. For the individual measurements of each chitooligosaccharide, PAPS was kept at a fixed, saturating concentration of 25 $\mu \mathrm{M}$. An average single-point normalization factor was determined to be 2.4 for chitobiose-6-OSO $\mathrm{O}_{3}^{-}, 3.0$ for chitotriose-6- $\mathrm{OSO}_{3}^{-}, 4.0$ for chitotetraose-6-OSO- , and 2.7 for chitopentaose-6-OSO$-\mathrm{O}_{3}^{-}$. The initial reaction velocity was calculated and plotted versus the chitooligosaccharide concentration, generating four saturation plots for the four acceptor substrates (Figure 1) (Figure 2a, b, $c, d)$. A nonlinear regression analysis of each saturation (a)

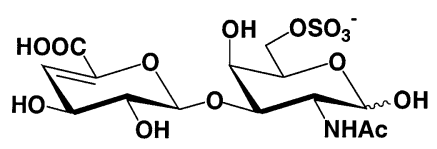

(b)

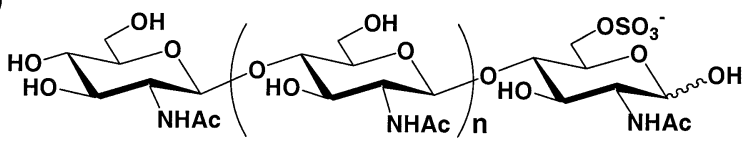

Figure 1. Structure of the internal standard and the four products monitored. (a) Internal standard, $\alpha-\Delta \mathrm{UA}-[1 \rightarrow 3]-G a l N A c-6 \mathrm{~S}$ $\left(\Delta \mathrm{D}_{\mathrm{i}}-6 \mathrm{~S}\right)$, with $\mathrm{m} / \mathrm{z}=458$; (b) Chitobiose-6-OSO ${ }_{3}^{-}(n=0)$ with $\mathrm{m} / \mathrm{z}$ $=503$, chitotriose-6-OSO ${ }_{3}^{-}(n=1)$ with $\mathrm{m} / \mathrm{z}=706$, chitotetraose6- $\mathrm{OSO}_{3}^{-}(n=2)$ with $\mathrm{m} / \mathrm{z}=909$, chitopentaose-6-OSO${ }_{3}^{-}(n=3)$ with $m / z=1112$.

plot was subsequently performed to obtain the kinetic constants. Average $K_{M}, k_{c a t}$, and $k_{c a t} / K_{M}$ values for four replicate analyses of chitobiose, chitotriose, chitotetraose, and chitopentaose are summarized in Table 1. There is an obvious 10 -fold decrease in the $K_{M}$ value from chitobiose to chitotriose. Chitotetraose, which has the lowest $K_{M}$ value of $14.9 \mu \mathrm{M}$, turns out to have the highest binding affinity to NodST among the four chitooligosaccharides. Although chitopentaose was revealed to have the highest reaction turn over number ( $k_{\text {cat }}$ value), chitotetraose was the most preferred substrate of NodST among the four, as indicated by the specificity constant values $\left(k_{c a t} / K_{M}\right)$. The $k_{\text {cat }} / K_{M}$ ratios were calculated to be 1:12:24:21 with respect to the $k_{\text {cat }} / K_{M}$ value of chitobiose. Chitobiose was arbitrarily chosen as the integer value because of its low specificity constant with respect to the other three chitooligomers. The relative reaction specificity of the four substrates determined by our assay was in excellent agreement with that reported in the literature [31, 37], which suggests that in the pathway of Nod signal biosynthesis, the lipochitotetrasaccharide Nod factor is preferably sulfated by NodH.

The method of elucidating the reaction specificity of NodH for multiple competing substrates by individual kinetic measurements was successfully performed using ESI-MS, as demonstrated above. However, a major problem of this technique is that all substrates must be studied individually and a group of varied concentrations of each substrate is necessary to obtain the specificity constant. In these individual measurements, seven reaction solutions were used for each of the four substrates tested and a total of 28 samples were generated. In order to radically simplify this procedure, a multiplex ESI-MS assay was developed and applied to NodST, in which multiple competing reactions of different substrates are handled in one reaction system to obtain specificity constants by ESI-MS analysis. 

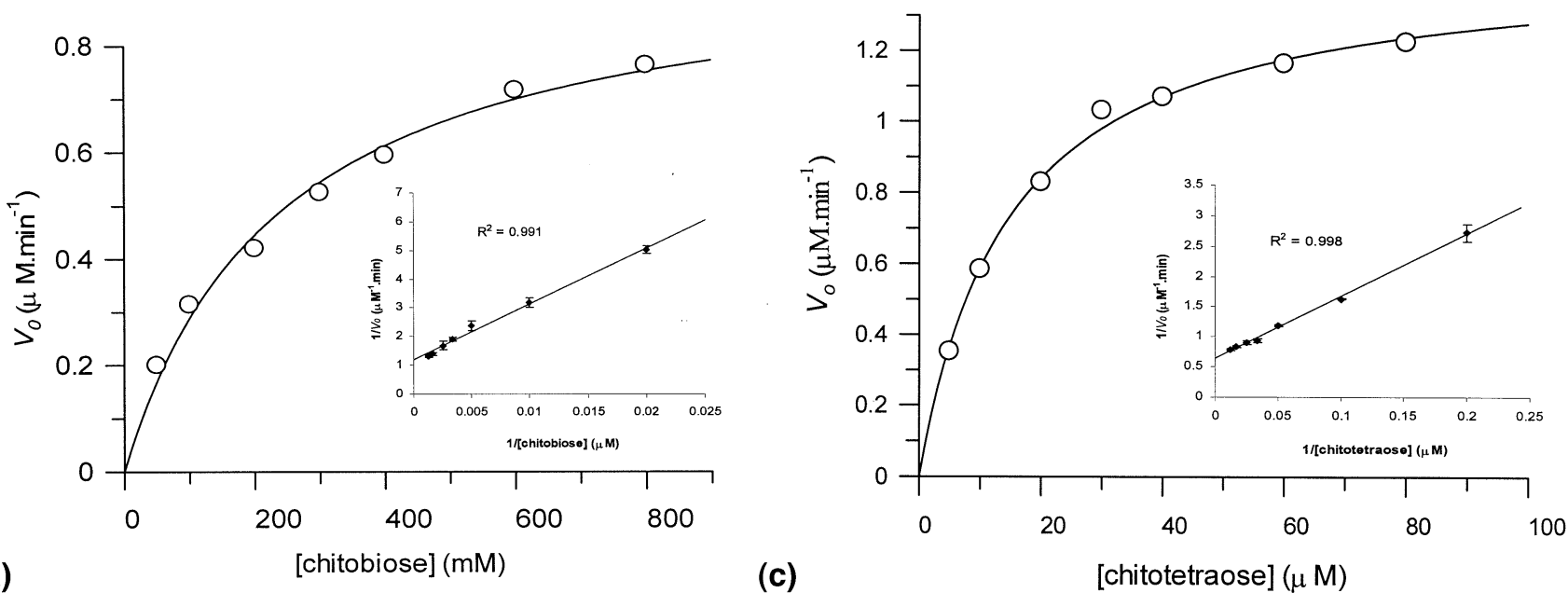

(c)

[chitotetraose] $(\mu \mathrm{M})$
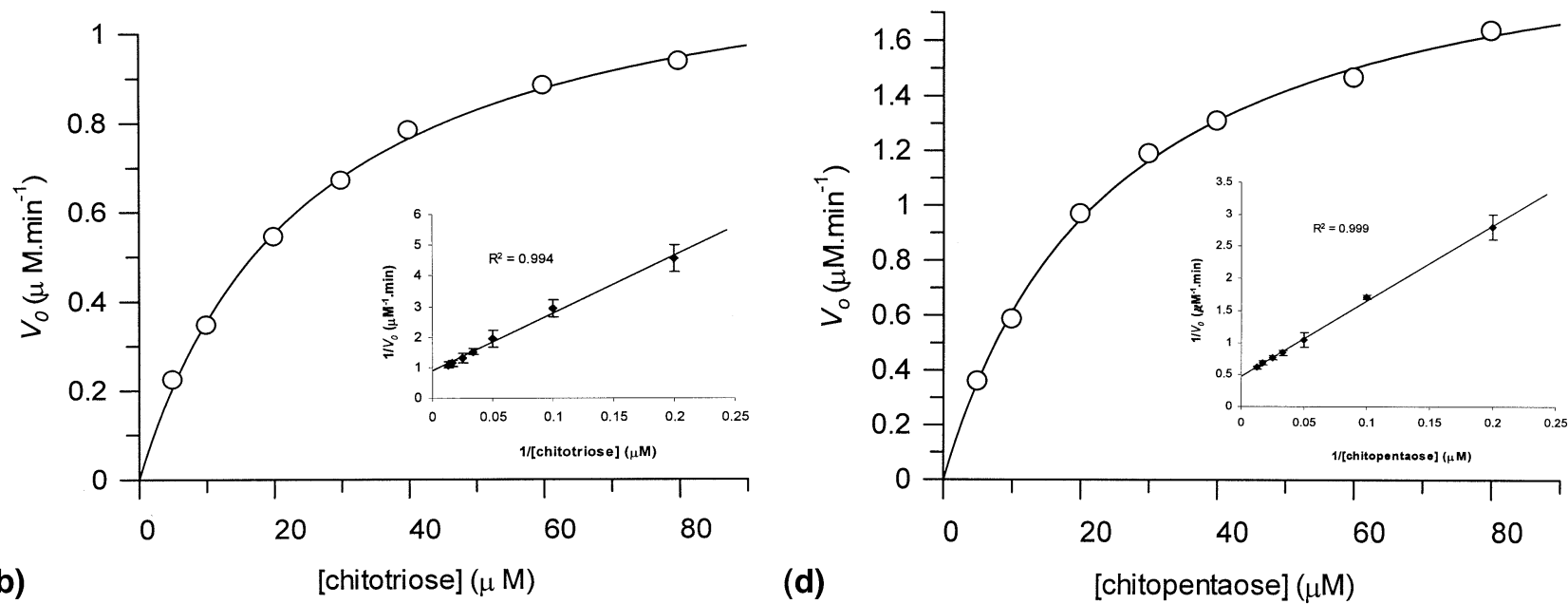

Figure 2. Determination of the kinetic constants for chitooligosaccharide substrates via individual kinetic measurements. (a) Saturation plot: $V_{0}$ vs. [chitobiose]. The inset is double reciprocal plot: $1 / V_{0}$ vs. $1 /$ [chitobiose]. ([Chitobiose] $=50,100,200,300,400,600,800 \mu \mathrm{M})$. (b) Saturation plot: $V_{0}$ vs. [chitotriose]. The inset is double reciprocal plot: $1 / V_{0}$ vs. $1 /$ [chitotriose]. ([Chitotriose] $=5,10,20,30$, $40,60,80 \mu \mathrm{M})$. (c) Saturation plot: $V_{0}$ vs. [chitotetraose]. The inset is double reciprocal plot: $1 / V_{0}$ vs. $1 /$ [chitotetraose]. ([Chitotetraose] $=5,10,20,30,40,60,80 \mu \mathrm{M})$. (d) Saturation plot: $V_{0}$ vs. [chitopentaose]. The inset is double reciprocal plot: $1 / V_{0}$ vs. $1 /$ [chitopentaose]. ([Chitopentaose] $=5$, $10,20,30,40,60,80 \mu \mathrm{M})$. [PAPS] was fixed at $25 \mu \mathrm{M}$ for all the four chitooligosaccharides. [NodST] $=50 \mathrm{nM}, \mathrm{pH}=8.0$. Each saturation plot was generated from the average data of three replicate experiments.

\section{Multiplex Substrate Specificity Evaluation}

A theoretical model for calculation. If several substrates are mixed together and compete for the same active site of an enzyme, the initial reaction rate of each competing substrate $\left(\nu_{n}\right)$ can be calculated using eq 3 ,

$$
\nu_{n}=\left(k_{c a t} / K_{M}\right)_{n} \cdot\left[S_{n}\right] /\left(1+\sum\left[S_{n}\right] /\left(K_{M}\right)_{n}\right)
$$

where $\left(K_{M}\right)_{n}$ and $\left(k_{\text {cat }} / K_{M}\right)_{n}$ are the Michaelis-Menten constant and specificity constant, respectively, for each particular substrate $\left(S_{n}\right)$.

Since the reaction rate is determined by more than just the concentrations and kinetic parameters of a single competing substrate, we can not obtain the
Table 1. Kinetic constants determined for chitooligosaccharide substrates in individual kinetic measurements

\begin{tabular}{lcccc}
\hline $\begin{array}{l}\text { Acceptor } \\
\text { substrate }\end{array}$ & $\begin{array}{c}K_{M} \\
(\mu \mathrm{M})\end{array}$ & $\begin{array}{c}k_{c a t} \\
\left(\mathrm{~min}^{-1}\right)\end{array}$ & $\begin{array}{c}k_{c a} / K_{M} \\
\left(\mu \mathrm{M}^{-1} \cdot \mathrm{min}^{-1}\right)\end{array}$ & $\begin{array}{c}\text { Relative } \\
k_{c a t} / K_{M}{ }^{c}\end{array}$ \\
\hline \hline Chitobiose & $240 \pm 10^{\mathrm{a}}$ & $19.5 \pm 0.7^{\mathrm{b}}$ & $0.082 \pm 0.005$ & 1 \\
Chitotriose & $25 \pm 5$ & $25 \pm 3$ & $1.0 \pm 0.1$ & $12 \pm 2$ \\
Chitotetraose & $14.9 \pm 0.2$ & $29 \pm 2$ & $2.0 \pm 0.1$ & $24 \pm 2$ \\
Chitopentaose & $23 \pm 4$ & $42 \pm 1$ & $1.7 \pm 0.1$ & $21 \pm 2$
\end{tabular}

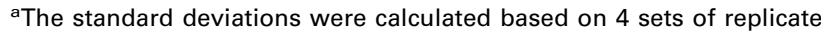
experiments for each substrate.

${ }^{\mathrm{b}}$ The $k_{c a t}$ values are calculated using the $V_{\max }$ value with NodST concentration of $50 \mathrm{nM}$.

${ }^{\mathrm{c}}$ The relative $k_{c a t} / K_{M}$ is represented by the ratios of $k_{c a t} / K_{M}$ values of the four substrates, which were calculated in respect to $k_{c a t} / K_{M}$ value of chitobiose. 
specificity constant of the substrate by solely plotting the initial rate versus the substrate concentration. However, the ratio of reaction rates for competing substrates in a mixture is related to their specificity constants and their concentrations, as shown in eq 4.

$$
\begin{gathered}
\nu_{1}: \nu_{2}: \ldots \ldots: \\
\nu_{n}=\left\{\left(k_{c a t} / K_{M}\right)_{1} \cdot\left[S_{1}\right]\right\}:\left\{\left(k_{c a t} / K_{M}\right)_{2}\right. \\
\left.\cdot\left[S_{2}\right]\right\}: \ldots \ldots:\left\{\left(k_{c a t} / K_{M}\right)_{n} \cdot\left[S_{n}\right]\right\}
\end{gathered}
$$

Hence, the specificity constant ratio of multiple competing substrates can be determined in one reaction using eq 5, given the initial reaction rate of each substrate.

$$
\begin{aligned}
& \left(k_{c a t} / K_{M}\right)_{1}:\left(k_{c a t} / K_{M}\right)_{2}: \ldots \ldots:\left(k_{c a t} / K_{M}\right)_{n} \\
& =\left(\nu_{1} /\left[S_{1}\right]\right):\left(\nu_{2} /\left[S_{2}\right]\right): \ldots \ldots:\left(\nu_{n} /\left[S_{n}\right]\right)
\end{aligned}
$$

In the multi-substrate reaction system, all the competing reactions are initiated and quenched at the same time and the reaction rates in eq 5 can be substituted by the corresponding product concentrations, resulting in eq 6.

$$
\begin{aligned}
& \left(k_{c a t} / K_{M}\right)_{1}:\left(k_{c a t} / K_{M}\right)_{2}: \ldots \ldots:\left(k_{c a t} / K_{M}\right)_{n} \\
& =\left(\left[P_{1}\right] /\left[S_{1}\right]\right):\left(\left[P_{2}\right] /\left[S_{2}\right]\right): \ldots \ldots:\left(\left[P_{n}\right] /\left[S_{n}\right]\right)
\end{aligned}
$$

Using mass spectrometry, the investigator has the advantage of monitoring and quantifying multiple species with different molecular weights without further separation, and multiple products of different molecular weights generated in the multi-substrate reaction system can be quantified simultaneously by monitoring the corresponding product peaks in relation to an internal standard peak. Since we know the starting concentration of each substrate and we can measure the concentration of each product, the $k_{c a t} / K_{M}$ ratios of multiple substrates can be easily determined and the substrate specificity of an enzyme can be rapidly evaluated. Therefore, as long as we know the $k_{\text {cat }} / K_{M}$ value of one substrate, the $k_{c a t} / K_{M}$ values for the rest of the competing substrates can be calculated using the $k_{\text {cat }} / K_{M}$ ratios.

Methodology for product quantification. In the individual kinetic measurements for each acceptor substrate, only one reaction was involved and one product was quantified. The product quantification was carried out using a single-point normalization factor, since there is a linear response between the concentration ratio and ion intensity ratio of the product being quantified and the internal standard. However, the multi-substrate reaction system contains multiple competing reactions and results in formation of multiple products, hence the parallel quantification of all the products is required. In particular, the linear responses between the concentration ratio and ion intensity ratio of all the products and the internal standard in the multi-substrate reaction system need to be carefully monitored. In order to check this, four calibration curves were constructed for the four sulfated chitooligosaccharide products in the multi-substrate reaction system of NodST. As demonstrated in Figure 3, excellent linearity $\left(R^{2}>0.99\right)$ was obtained for all four calibration curves over a wide range of product and internal standard concentration ratios $(0.02$ to 1$)$, and the lowest product concentration tested in each calibration curve was $0.1 \mu \mathrm{M}$. The results strongly indicate a linear response between the concentration ratio and ion intensity ratio of each product and the internal standard. Furthermore, this linear response is not affected by the presence of other substrates and products in the sample. Thus, the quantification of multiple products formed in the multi-substrate reaction system is feasible. The slope of each calibration curve was determined to be 2.8 for chitobiose- $6-\mathrm{OSO}_{3}^{-}$, 4.0 for chitotriose-6- $\mathrm{OSO}_{3}^{-}, 4.0$ for chitotetraose-6$\mathrm{OSO}_{3}^{-}$, and 3.1 for chitopentaose-6-OSO - , which were subsequently used as the normalization factors to quantify the corresponding product in the multi-substrate reaction system.

Specificity constant $\left(k_{\mathrm{cat}} / K_{\mathrm{M}}\right)$ measurement and substrate specificity evaluation. Since the specificity constant value of chitobiose is one order of magnitude lower than the other three acceptor substrates, as revealed in our individual kinetic measurements, we adjusted the starting concentration of chitobiose $(300 \mu \mathrm{M})$ to be ten times the concentration of the other three $(30 \mu \mathrm{M})$ in the multi-substrate reaction system to facilitate quantification.

As shown in Figure 4, five peaks in the quenched reaction sample were monitored simultaneously using the SIM mode of ESI-MS. They are the internal standard peak at $\mathrm{m} / \mathrm{z} 458$, and the four sulfated chitooligosaccharide peaks at $m / z 503,706,909$, and 1112. Parallel quantification of the four sulfated products was performed at different reaction times to generate the reaction progress curves, which indicated that following two minutes of reaction time the reaction conversion for chitobiose, chitotriose, chitotetraose, and chitopentaose were $0.1 \%, 1.5 \%, 3.5 \%$, and $2.4 \%$, respectively. Since the sulfation percentage of all the substrates was lower than $5 \%$ after $2 \mathrm{~min}$ of reaction, a reaction time of $2 \mathrm{~min}$ should reside in the initial period of all the four reactions. It is important to note that to obtain the correct specificity constant ratios using eq 6 , the product concentrations at the initial reaction stage must be used. This is a direct result of the conditions required in eq 3 , which describes the initial reaction velocity of each competing substrate in a multi-substrate enzymatic system, from which eq 6 is derived. Hence, the product concentrations at a two minute reaction were used to calculate the $k_{c a t} / K_{M}$ ratios of the four acceptor sub- 

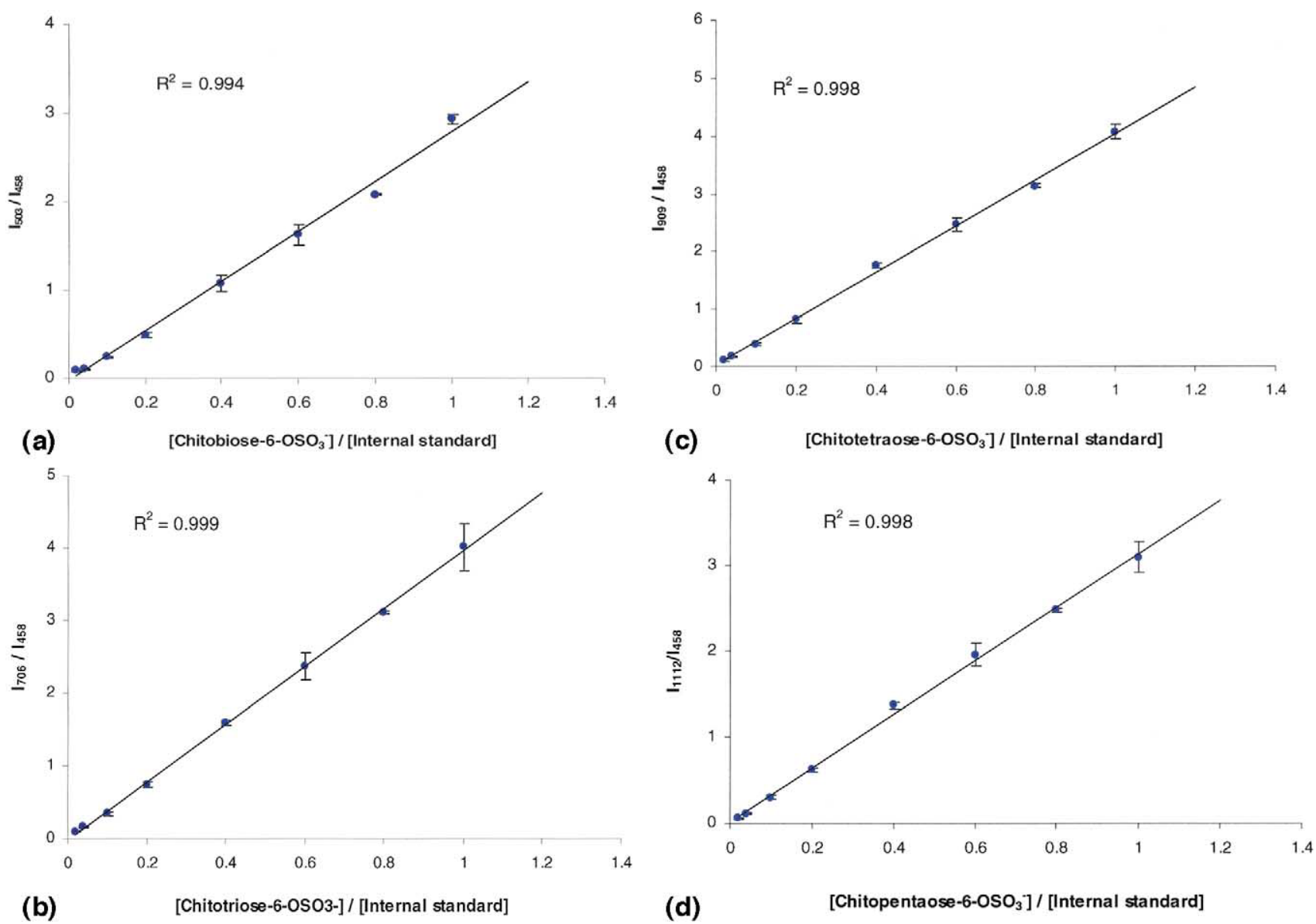

Figure 3. Calibration curves for the four sulfated chitooligosaccharide products in the multisubstrate reaction system. (a) $\mathrm{I}_{503} / \mathrm{I}_{458}$ vs. [chitobiose-6-OSO - ] / [internal standard]. (b) $\mathrm{I}_{706} / \mathrm{I}_{458}$ vs. [chitotriose-6-OSO $-\mathrm{OSO}_{3}^{-}$]/[internal standard]. (c) $\mathrm{I}_{909} / \mathrm{I}_{458} \mathrm{vs}$. [chitotetraose-6-OSO - ]/[internal standard]. (d) $\mathrm{I}_{1112} / \mathrm{I}_{458}$ vs. [chitopentaose-6-OSO - ]/[internal standard]. A mixed completed reaction solution was used for calibration curve construction, in which [PAPS] $=50 \mu \mathrm{M}$, [chitobiose-6- $\mathrm{OSO}_{3}^{-}$] $=$ $\left[\right.$ chitotriose-6-OSO $\left.{ }_{3}^{-}\right]=\left[\right.$chitotetraose- $\left.6-\mathrm{OSO}_{3}^{-}\right]=\left[\right.$chitopentaose-6-OSO $\left.\mathrm{O}_{3}^{-}\right]=25 \mu \mathrm{M}$. [NodST $]=50$ $\mathrm{nM}, \mathrm{pH}=8.0$. Each calibration curve was generated from the average data of three replicate experiments.

strates based on the theoretical calculation model. The average results of three replicate experiments are listed in the first column of Table 2, which are in good agreement with the ratios obtained from the individual measurements (Table 1). The consistency of the results strongly supports the fact that the multiplex ESI-MS assay, based on the theoretical steady-state kinetics model, is valid for accurate enzyme specificity determination with multiple competing substrates. Given the $k_{c a t} / K_{M}$ ratios of the four substrates obtained in the multiplex assay, and having the $k_{\text {cat }} / K_{M}$ value for any one substrate determined in the individual measurement, we can subsequently calculate the $k_{\text {cat }} / K_{M}$ values for all the other substrates. As listed in the second column of Table 2, the $k_{\text {cat }} / K_{M}$ values of chitotriose, chitotetraose, and chitopentaose were calculated based on the $k_{c a t} / K_{M}$ value of chitobiose obtained in the individual study.

For substrate specificity studies, our multiplex assay is clearly competitive with the traditional methodology.
One main advantage is that only one reaction system and one quenched sample is required: all the substrates tested are mixed together and their specificity information can be rapidly obtained in only one measurement. Another important advantage of the multiplex assay is that small substrate concentrations $\left(\leq 0.2 K_{M}\right)$, which are necessary in the individual kinetic measurement to generate accurate saturation plot, are not required in this new methodology. Since a small starting concentration of substrate will result in formation of very small amount of product, the instrument needs to have a high sensitivity and low detection limit to achieve accurate product quantification. However, in the multiplex assay, there are no specific requirements for the starting concentration of each substrate. A considerable amount of each product will be formed in the multi-substrate reaction system when all the competing reactions are quenched in the initial reaction period. Therefore, accurate specificity constant ratios can be obtained by quantifying multiple products. 


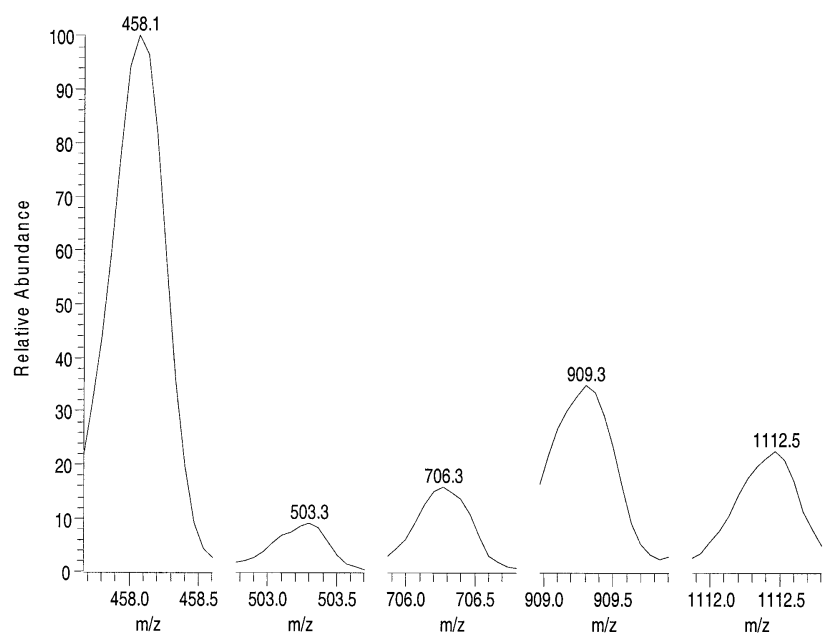

Figure 4. Selected ion monitoring (SIM) of five peaks in the multi-substrate reaction system. The internal standard peak is at $\mathrm{m} / \mathrm{z} 458$ and the four product peaks are at $\mathrm{m} / \mathrm{z} 503,706,909,1112$. [PAPS $]=25 \mu \mathrm{M}$, [chitobiose $]=300 \mu \mathrm{M}$, [chitotriose $]=$ [chitotetraose $]=[$ chitopentaose $]=30 \mu \mathrm{M}$. [NodST $]=50 \mathrm{nM}, \mathrm{pH}=8.0$.

\section{Conclusions}

A novel multiplex ESI-MS assay for determining specificity constants was developed and applied to the study of NodST, a GlcNAc-6-O-sulfotransferase. A substrate library consisting of four chitooligosaccharides (chitobiose, chitotriose, chitotetraose, and chitopentaose) was used, and the specificity constant $\left(k_{\text {cat }} / K_{M}\right)$ of each library component was first determined by individual kinetic measurements. Subsequently, a multiplex assay was used to rapidly determine the specificity constant ratios for a mixture of the four chitooligosaccharide substrates. The specificity constant ratios determined from the multiplex assay were found to be in
Table 2. Substrate specificity evaluation and specificity constant measurement using the multiplex ESI-MS assay

\begin{tabular}{lcc}
\hline Acceptor substrate & $\begin{array}{c}\text { Relative } \\
k_{c a t} / K_{M}{ }^{\mathrm{a}}\end{array}$ & $\begin{array}{c}k_{c a t} / K_{M} \\
\left(\mu \mathrm{M}^{-1} \cdot \mathrm{min}^{-1}\right)\end{array}$ \\
\hline \hline Chitobiose & 1 & 0.082 \\
Chitotriose & $12.3 \pm 0.2^{\mathrm{b}}$ & $1.0 \pm 0.0$ \\
Chitotetraose & $27.6 \pm 0.6$ & $2.3 \pm 0.1$ \\
Chitopentaose & $21 \pm 2$ & $1.8 \pm 0.2$ \\
\hline
\end{tabular}

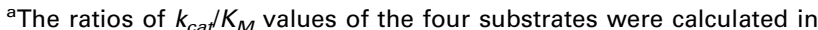
respect to chitobiose.

bThe standard deviations were calculated based on 3 sets of replicate experiments for each substrate.

good agreement with those obtained from the individual kinetic measurements. The consistency of the results demonstrated the multiplex ESI-MS assay to be an accurate and valid method for substrate specificity evaluation of NodST. Using this assay, multiple substrates of different sizes can be tested in one reaction system and their reaction specificity can be revealed by one measurement in which all the products are quantified simultaneously. Compared with the traditional method for substrate specificity study, this new assay requires fewer experimental materials, shorter analysis time, and is expected to be an efficient and promising methodology to study other enzymes of therapeutic interest. Current efforts are focused on applying this multiplex methodology to the specificity study of a synthetic substrate library of HS sulfotransferase.

\section{Acknowledgments}

The authors gratefully acknowledge the National Institute of Health (NIH), GM63521 for financial support. The authors also thank the American Society of Mass Spectrometry (ASMS) for allowing them to present this research at the 51st ASMS Conference, Montreal, Canada, in June, 2003. 


\section{Appendix}

\section{Supporting information: Calibration curves for each sulfated chitooligosaccharide product in the single substrate reaction system}

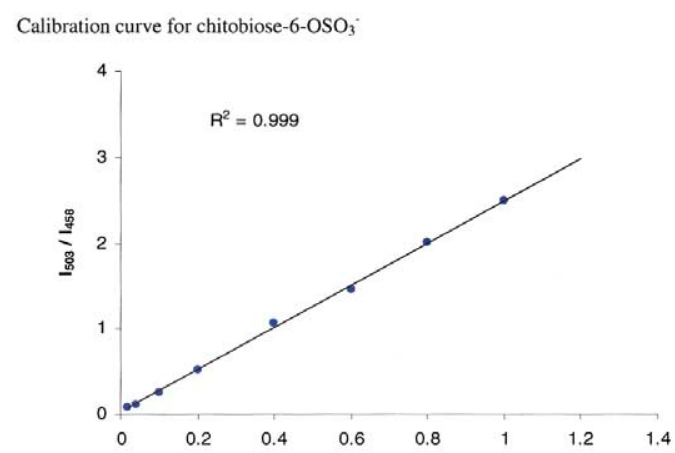

(a)

[Chitobiose-6- $\mathrm{OSO}_{3}$ ] / [Internal standard]

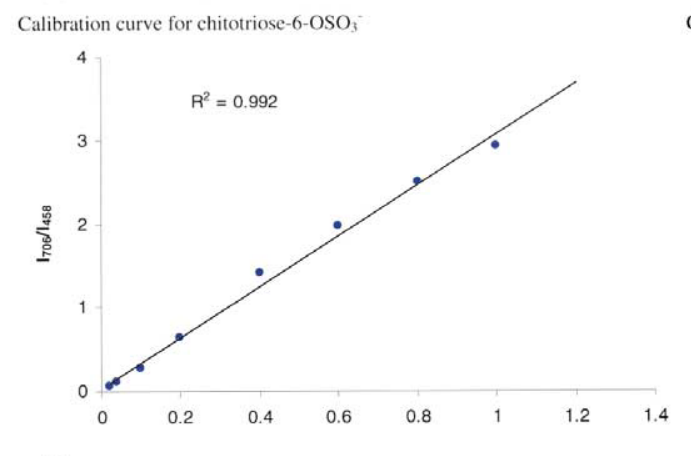

(b)

[Chitotriose-6- $\left.-\mathrm{OSO}_{3}\right] /[$ Internal standard]

\section{References}

1. Usami, N.; Yamamoto, T.; Shintani, S.; Higaki, Y.; Ishikura, S.; Katagiri, Y.; Hara, A. Substrate Specificity of Human 3(20) $\alpha-$ Hydroxysteroid Dehydrogenase for Neurosteroids and Its Inhibition by Benzodiazepines. Biol. Pharm. Bull. 2002, 25, 441-445.

2. Kelly, B. S.; Antholine, W. E.; Griffith, O. W. Escherichia coli $\gamma$-Glutamylcysteine Synthetase-Two Active Site Metal Ions Affect Substrate and Inhibitor Binding. J. Biol. Chem. 2002, 277, $50-58$.

3. Freel Meyers, C. L.; Oberthur, M.; Anderson, J. W.; Kahne, D.; Walsh, C. T. Coumarin Formation in Novobiocin Biosynthesis: $\beta$-Hydroxylation of the Aminoacyl Enzyme Tyrosyl-S-NovH by a Cytochrome P450 NovI. Chem. Biol. 2001, 8, 301-312.

4. Siviter, R. J.; Nachman, R. J.; Dani, M. P.; Keen, J. N.; Shirras, A. D.; Isaac, R. E. Peptidyl Dipeptidases (Ance and Acer) of Drosophila melanogaster: Major Differences in the Substrate Specificity of Two Homologs of Human Angiotensin I-Converting Enzyme. Peptides 2002, 23, 2025-2034.

5. Rong, J.; Habuchi, H.; Kimata, K.; Lindahl, U.; Kusche-Gullberg, M. Substrate Specificity of the Heparan Sulfate Hexuronic Acid 2-O-Sulfotransferase. Biochemistry 2001, 40, 55485555.

6. Habuchi, H.; Tanaka, M.; Habuchi, O.; Yoshida, K.; Suzuki, H.; Ban, K.; Kimata, K. The Occurrence of Three Isoforms of Heparan Sulfate 6-O-Sulfotransferase Having Different Specificities for Hexuronic Acid Adjacent to the Targeted NSulfoglucosamine. J. Biol. Chem. 2000, 275, 2859-2868.

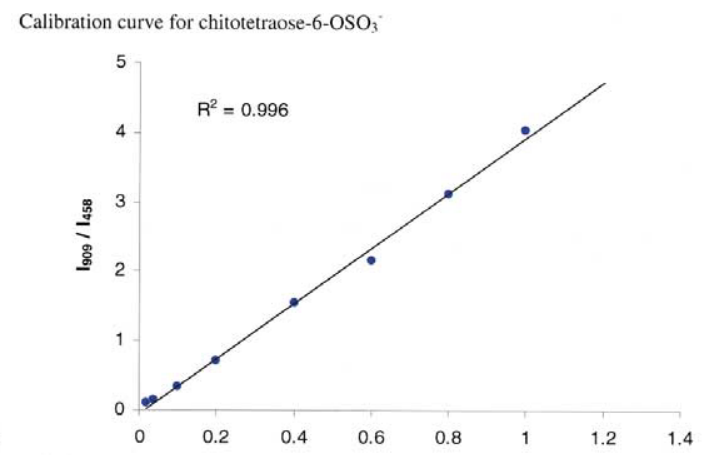

(c)

[Chitotetraose-6-0 $\mathrm{OSO}_{3}$ ] / [Internal standard]

Calibration curve for chitopentaose-6- $\mathrm{OSO}_{3}$

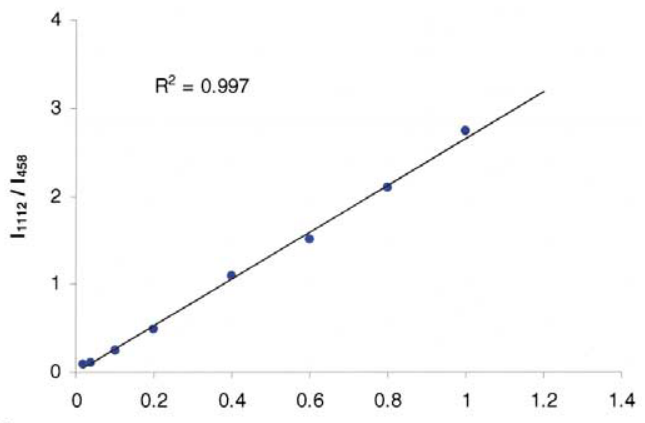

(d)

[Chitopentaose-6-OSO $\left.{ }_{3}\right] /[$ Internal standard]

7. Dajani, R.; Cleasby, A.; Neu, M.; Wonacott, A. J.; Jhoti, H.; Hood, A. M.; Modi, S.; Hersey, A.; Taskinen, J.; Cooke, R. M.; Manchee, G. R.; Coughtrie, M. W. H. X-ray Crystal Structure of Human Dopamine Sulfotransferase, SULT1A3: Molecular Modeling and Quantitative Structure-Activity Relationship Analysis Demonstrate a Molecular Basis for Sulfotransferase Substrate Specificity. J. Biol. Chem. 1999, 274, 37862-37868.

8. Qian, Y.-M.; Song, W.-C. Correlation Between PAP-Dependent Steroid Binding Activity and Substrate Specificity of Mouse and Human Estrogen Sulfotransferases. J. Steroid Biochem. 1999, 71, 123-131.

9. Martins, L. L.; Mourato, M. P.; De Varennes, A. Effects of Substrate Structural Analogues on the Enzymatic Activities of Aspartate Aminotransferase Isoenzymes. J. Enzym. Inhib. 2001, 16, 251-257.

10. Chen, H. W.; Walsh, C. T. Coumarin Formation in Novobiocin Biosynthesis: $\beta$-Hydroxylation of the Aminoacyl Enzyme Tyrosyl-S-NovH by a Cytochrome P450 NovI. Chem. Biol. 2001, 8, 301-312.

11. Losey, H. C.; Peczuh, M. W.; Chen, Z.; Eggert, U. S.; Dong, S. D.; Pelczer, I.; Kahne, D.; Walsh, C. T. Tandem Action of Glycosyltransferases in the Maturation of Vancomycin and Teicoplanin Aglycones: Novel Glycopeptides. Biochemistry 2001, 40, 4745-4755.

12. Corbett, K.; Fordham-Skelton, A. P.; Gatehouse, J. A.; Davis, B. G. Tailoring the Substrate Specificity of the $\beta$-Glycosidase from the Thermophilic Archaeon Sulfolobus solfataricus. FEBS Lett. 2001, 509, 355-360. 
13. Walsh, C. T.; Chen, H. W.; Keating, T. A.; Hubbard, B. K.; Losey, H. C.; Luo, L. S.; Marshall, C. G.; Miller, D. A.; Patel, H. M. Tailoring Enzymes that Modify Nonribosomal Peptides During and After Chain Elongation on NRPS Assembly Lines. Curr. Opin. Chem. Biol. 2001, 5, 525-534.

14. Marshall, A. D.; Darbyshire, J. F.; Hunter, A. P.; McPhie, P.; Jakoby, W. B. Control of Activity Through Oxidative Modification at the Conserved Residue Cys(66) of Aryl Ssulfotransferase IV. J. Biol. Chem. 1997, 272, 9153-9160.

15. Matsumoto, K.; Davis, B. G.; Jones, J. B. Glycosylation of the Primary Binding Pocket of a Subtilisin Protease Causes a Remarkable Broadening in Stereospecificity in Peptide Synthesis. Chem. Commun. 2001, 10, 903-904.

16. Ichiyama, S.; Kurihara, T.; Li, Y.-F.; Kogure, Y.; Tsunasawa, S.; Esaki, N. Novel Catalytic Mechanism of Nucleophilic Substitution by Asparagine Residue Involving Cyanoalanine Intermediate Revealed by Mass Spectrometric Monitoring of an Enzyme Reaction. J. Biol. Chem. 2000, 275, 40804-40809.

17. Pi, N., Yu, Y., Mougous, J. D., Leary, J. A. Observation of a Hybrid Random Ping-Pong Mechanism of Catalysis for NodST: A Mass Spectrometry Approach, unpublished.

18. Vocadlo, D. J.; Wicki, J.; Rupitz, K.; Withers, S. G. A Case for Reverse Protonation: Identification of Glu160 as an Acid/Base Catalyst in Thermoanaerobacterium saccharolyticum ss-Xylosidase and Detailed Kinetic Analysis of a Site-Directed Mutant. Biochemistry 2002, 41, 9736-9746.

19. Imamura, H.; Fushinobu, S.; Jeon, B. S.; Wakagi, T.; Matsuzawa, H. Identification of the Catalytic Residue of Thermococcus litoralis 4- $\alpha$-Glucanotransferase Through MechanismBased Labeling. Biochemistry 2001, 40, 12400-12406.

20. Recksiek, M.; Selmer, T.; Dierks, T.; Schmidt, B.; Von Figura, K. Sulfatases, Trapping of the Sulfated Enzyme Intermediate by Substituting the Active Site Formylglycine. J. Biol. Chem. 1998, 273, 6096-6103.

21. Vocadlo, D. J.; Davies, G. J.; Laine, R.; Withers, S. G. Catalysis by Hen Egg-White Lysozyme Proceeds via a Covalent Intermediate. Nature 2001, 412, 835-838.

22. Rhomberg, A. J.; Shriver, Z.; Biemann, K.; Sasisekharan, R. Mass Spectrometric Evidence for the Enzymatic Mechanism of the Depolymerization of Heparin-Like Glycosaminoglycans by Heparinase II. Proc. Natl. Acad. Sci. U.S.A. 1998, 95, 12232 12237.

23. Houston, C. T.; Taylor, W. P.; Widlanski, T. S.; Reilly, J. P. Investigation of Enzyme Kinetics Using Quench-Flow Techniques with MALDI TOF Mass Spectrometry. Anal. Chem. 2000, 72, 3311-3319.

24. Hsieh, F. Y. L.; Tong, X.; Wachs, T.; Ganem, B.; Henion, J. Kinetic Monitoring of Enzymatic Reactions in Real-Time by Quantitative High-Performance Liquid-Chromatography Mass Spectrometry. Anal. Biochem. 1995, 229, 20-25.

25. Newton, R. P.; Bayliss, M. A.; Khan, J. A.; Bastani, A.; Wilkins, A. C. R.; Games, D. E.; Walton, T. J.; Brenton, A. G.; Harris, F. M. Kinetic Analysis of Cyclic CMP-Specific and Multifunc- tional Phosphodiesterases by Quantitative Positive-Ion FastAtom Bombardment Mass Spectrometry. Rapid Commun. Mass Spectrom. 1999, 13, 574-584.

26. Bothner, B.; Chavez, R.; Wei, J.; Strupp, C.; Phung, Q.; Schneemann, A.; Siuzdak, G. Monitoring Enzyme Catalysis with Mass Spectrometry. J. Biol. Chem. 2000, 275, 13455-13459.

27. Ge, X.; Sirich, T. L.; Beyer, M. K.; Desaire, H.; Leary, J. A. A Strategy for the Determination of Enzyme Kinetics Using Electrospray Ionization with an Ion Trap Mass Spectrometer. Anal. Chem. 2001, 73, 5078-5082.

28. Gao, H.; Leary, J. A. Multiplex Inhibitor Screening and Kinetic Constant Determinations for Yeast Hexokinase Using Mass Spectrometry Based Assays. J. Am. Soc. Mass. Spectrom. 2003, 14, 173-181.

29. Pi, N.; Armstrong, J. I.; Bertozzi, C. R.; Leary, J. A. Kinetic Analysis of NodST Sulfotransferase Using an Electrospray Ionization Mass Spectrometry Assay. Biochemistry 2002, 41, 13283-13288.

30. Ehrhardt, D. W.; Atkinson, E. M.; Faull, K. F.; Freedberg, D. I.; Sutherlin, D. P.; Armstrong, R.; Long, S. R. in vitro Sulfotransferase Activity of NodH, a Nodulation Protein of Rhizobium meliloti Required for Host-Specific Nodulation. J. Bacteriol. $1995,177,6237-6245$.

31. Schultze, M.; Staehelin, C.; Rohrig, H.; John, M.; Schmidt, J.; Kondorosi, E.; Schell, J.; Kondorosi, A. in vitro Sulfotransferase Activity of Rhizobium meliloti NodH Protein-Lipochitooligosaccharide Nodulation Signals are Sulfated After Synthesis of the Core Structure. Proc. Natl. Acad. Sci. U.S.A. 1995, 92, 2706-2709.

32. Atkinson, E. M.; Palcic, M. M.; Hindsgaul, O.; Long, S. R. Biosynthesis of Rhizobium meliloti Lipooligosaccharide Nod Factors-NodA is Required for an N-Acyltransferase Activity. Proc. Natl. Acad. Sci. U.S.A. 1994, 91, 8418-8422.

33. Roche, P.; Debelle, F.; Maillet, F.; Lerouge, P.; Faucher, C.; Truchet, G.; Denarie, J.; Prome, J. C. Molecular Basis of Symbiotic Host Specificity in Rhizobium meliloti: nodH and nodPQ Genes Encode the Sulfation of Lipo-Oligosaccharide Signals. Cell 1991, 67, 1131-1143.

34. Truchet, G.; Roche, P.; Lerouge, P.; Vasse, J.; Camut, S.; De Billy, F.; Prome, J. C.; Denarie, J. Sulfated Lipo-Oligosaccharide Signals of Rhizobium meliloti Elicit Root Nodule Organogenesis in Alfalfa. Nature 1991, 351, 670-673.

35. Samain, E.; Chazalet, V.; Geremia, R. A. Production of $O-$ Acetylated and Sulfated Chitooligosaccharides by Recombinant Escherichia coli Strains Harboring Different Combinations of nod genes. J. Biotechnol. 1999, 72, 33-47.

36. Southwick, A. M.; Wang, L. X.; Long, S. R.; Lee, Y. C. Activity of Sinorhizobium meliloti NodAB and NodH Enzymes on Thiochitooligosaccharides. J. Bacteriol. 2002, 184, 4039-4043.

37. Burkart, M. D.; Izumi, M.; Chapman, E.; Lin, C. H.; Wong, C. H. Regeneration of PAPS for the Enzymatic Synthesis of Sulfated Oligosaccharides. J. Org. Chem. 2000, 65, 5565-5574. 